\title{
Anticancer Therapeutics Decorated Nanomaterials
}

\author{
Ignác Capek* \\ Slovak Academy of Sciences, Institute of Measurement Science, Bratislava, Slovakia
}

*Corresponding author: Ignác Capek, Slovak Academy of Sciences, Institute of Measurement Science, Bratislava, Slovakia.

Received Date: February 03, 2020

Published Date: February 18, 2020

\begin{abstract}
The cause of cancer diseases is dependent on various internal and external factors including inherited immune conditions, and genetic mutations and unhealthy diet, tobacco-chewing and smoking habits, and infections, respectively. Nanoscale drug delivery platforms have shown promising clinical results in several types of cancer and inflammatory disorders. Nanoparticles have special characteristics of high surface to volume ratio, size controllability, and can be easily functionalized during synthesis. Cytotoxic therapeutics adsorbed to nanoparticles are used during hormone therapy, immunotherapy, chemotherapy, etc. which preferentially kill the rapidly dividing cancer cells while minimizing the damage to the normal tissue. The toxic core of inorganic nanoparticles is protected by providing a physical barrier (functional polymers) between the core and the external environment. After linking to poly (ethylene glycol) molecules, the polymer-coated toxic dots did not change their optical and colloidal properties over a broad range of external environment conditions. This spontaneous encapsulation process removes the problems of particle aggregation in physiological buffers.
\end{abstract}

Keywords: Cancer; Tumours, Nanoparticles; Therapeutics; Angiogenesis; Cellular uptake

\section{Introduction}

Cancer is a group of associated diseases which involved abnormal cell growth possessing the potential to intrude or spread to other parts of the body. It is believed to be one of the most grave and fatal diseases responsible for large number of deaths worldwide. The cause of cancer diseases is dependent on various internal and external factors including inherited immune conditions, and genetic mutations and unhealthy diet, tobaccochewing habit, and infections, respectively. These factors may act together or in sequence to cause cancer. However, a careful consideration of our diet, and prevention of heavy consumption of alcohol and tobacco use, may curb the chances of cancer diseases completely. Furthermore, avoiding excessive sun exposure and indoor tanning devices may prove to be fruitful in skin cancer and which could save millions skin cancer patients. On the other hand, substantial proportion of certain cancers caused by infectious agents, such as human papillomavirus (HPV), hepatitis B virus (HBV), hepatitis C virus (HCV), human immunodeficiency virus (HIV), and Helicobacter pylori (H. pylori) could be evaded through vaccination, or by treating the infection. By definition, cancer is the uncontrolled growth of abnormal cells which is ultimately governed by deregulation of growth factor signaling networks. Colony- stimulating factors (CSF), epidermal growth factor (EGF), fibroblast growth factor (FGF) hepatocyte growth factor (HGF), hepatoma-derived growth factor (HDGF), insulin, insulin-like growth factors (IGFs), interleukins, platelet-derived growth factor (PDGF), transforming growth factors (TGFs), tumor necrosis factoralpha (TNF- $\alpha$ ), and vascular endothelial growth factor (VEGF) are some of the growth factors that control proliferation, cell cycle progression, cellular differentiation, and apoptosis when interact with their respective receptors. Receptors of growth factors are often tyrosine kinase proteins which get activated upon binding with specific ligands through auto-phosphorylation.

\section{Discussion}

Nanoparticles used in medicine represent a broad range of materials, including liposomes, polymeric micelles, dendrimers, superparamagnetic iron oxide particles, quantum dots, metal nanoparticles (MNPs), rare earth doped NPs, polymer, silica, and carbon NPs, dendrimers, block copolymer micelles, lyposomes, etc. can be decorated with various drugs and (chemo)therapeutics. The nanoparticles synthesized by both "top down" and "bottom up" approaches are then functionalized with bioagents [1]. They include the oil-in-water microemulsion, double-emulsion, surfactant-mediated hydrothermal synthesis, nanoprecipitation, etc. Nanomaterials show unique properties such as high surface 
areas, tunable pore sizes, high concentration of surface-active groups, amorphous and crystalline domains, large pore volumes. The functionalization of nanoparticles designs and prepares desired structures with low toxicity, high stability, favorable drug release profiles and acceptable cellular uptake. Porous silica nanoparticles (PSiNPs), for example, has already been shown in the field of drug delivery [2]. The researchers have functionalized their surface to further improve the biological and physicochemical properties of the NPs for efficient intracellular drug delivery. Various ligands can be either directly incorporated in the porous silica matrix or grafted to the outer surface of the nanoparticles. These NPs can be decorated with some agents in several ways, including loading of cargo into the pores, covalent grafting, co-condensation of siloxyderived cargoes, etc.

The vasculature in tumours is known to beleaky to nanoparticles. The lymph system of tumours is poorly operational and particles leaking from the blood vessels accumulate - a phenomenon known as "enhanced permeability and retention (EPR) effect" [3]. It has been shown that entities in the order of hundreds of nanometers in size can leak out of the blood vessels and accumulate within tumours. However, large nanoparticles could have limited diffusion in the extracellular space [4]. Experiments from animal models suggest that sub-150 nm, neutral or slightly negatively charged entities can move through tumour tissue [5]. Additionally, some data show that nanoparticles in the 50-100 $\mathrm{nm}$ size range that carry a very slight positive charge can penetrate throughout large tumours following systemic administration [6]. Thus, well designed nanoparticles in the 10-100 nm size range and with a surface charge either slightly positive or slightly negative should have accessibility to and within disseminated tumours when dosed into the circulatory system. If this size range is correct, then these nanoparticles will be restricted from exiting normal vasculature (that requires sizes less than 1-2 $\mathrm{nm}$ ); however they will still be able to access the liver, as entities up to $100-150 \mathrm{~nm}$ in diameter are able to do so.

The term angiogenesis is generally applied to the growth of micro vessel sprouts the size of capillary blood vessels, a process that is orchestrated by a range of angiogenic factors. Although proliferating endothelial cells undergoing DNA synthesis are a common hallmark of angiogenic microvascular sprouts, extensive sprouts can grow for periods of time, mainly by the migration of endothelial cells [7]. Physiological angiogenesis is distinct from arteriogenesis and lymph angiogenesis and occurs in reproduction, development and wound repair. It is usually focal, such as in blood coagulation in a wound, and self-limited in time, taking days (ovulation), weeks (wound healing) or months (placentation). By contrast, pathological angiogenesis can persist for years. Pathological angiogenesis is necessary for tumours and their metastases to grow beyond a microscopic size and it can give rise to bleeding, vascular leakage and tissue destruction. These consequences of pathological angiogenesis can be responsible, directly or indirectly, for the symptoms, incapacitation or death associated with a broad range of 'angiogenesis-dependent diseases' [8]. Examples of such diseases include cancer, autoimmune diseases, age-related macular degeneration and atherosclerosis. This process has been the subject of intense research due to its role in cancer development and has proven to be the result of numerous interactions between regulators, mediators and stimulatory molecules. These molecules regulate the proliferative and invasive activity of the endothelial cells that line blood vessels. Some of the most prominent angiogenesis stimulatory molecules include vascular endothelial growth factor (VEGF), basic fibroblast growth factor, platelet-derived growth factor and certain matrix metalloproteinases (Figure 1).

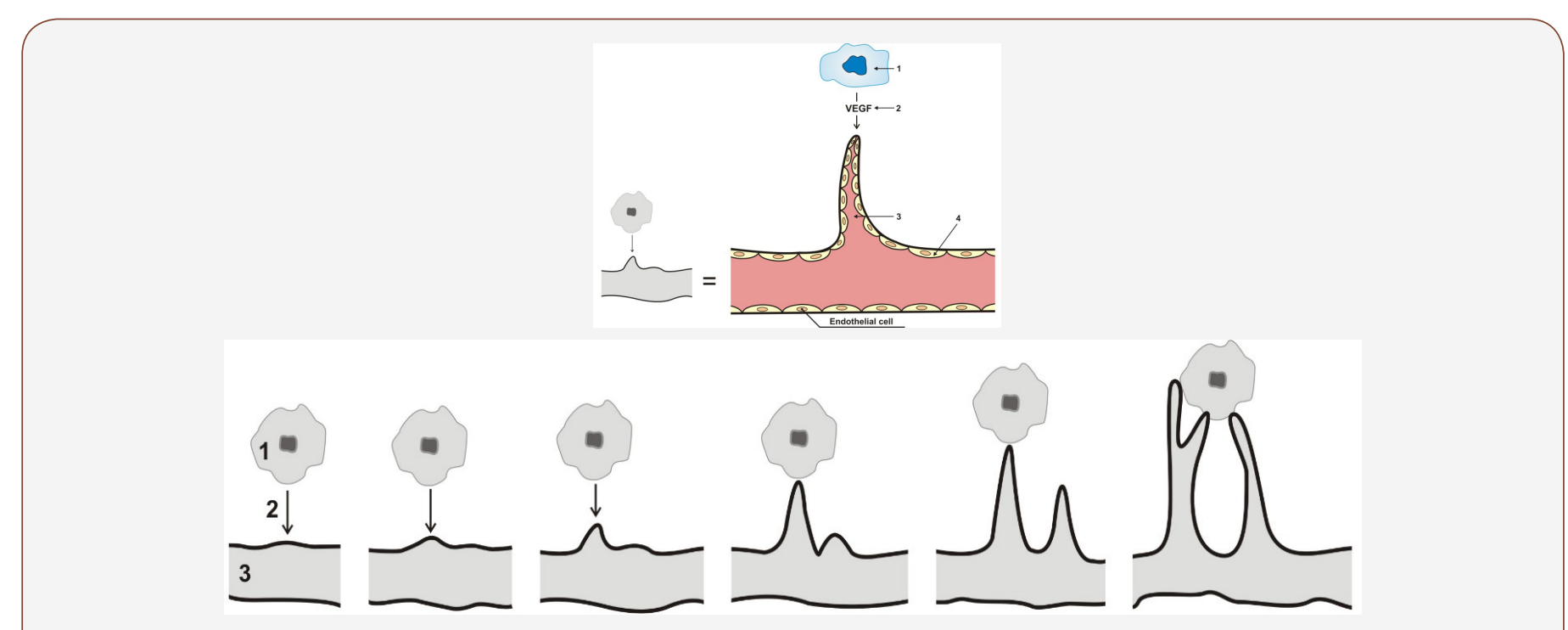

Figure 1: Angiogenesis and vascularization mechanisms; tumour expression (1) of an angiogenic factor, an angiogenic factor after its secretion from a tumour and an endothelial cell receptor. VEGF (2), vascular endothelial growth factor. Continued tumor development beyond diffusionlimited maximal size. In (B), the angiogenic switch has occurred creating an imbalance of positive to negative regulators causing endothelial cell proliferation and migration. These endothelial cells (4) form a vessel which extends towards the tumor and provides nutrients to sustain cell proliferation. A fully vascularized tumor is capable of continued growth with metastatic potential due to the proximity to the blood stream (3) [9]. 
Passive targeting of nanoparticles relies upon the unique pharmacokinetics of nanoparticles including minimal renal clearance and the "enhanced permeability and retention" (EPR) effect in the tumor. Angiogenesis and vascularization are wellcharacterized for tumors [10]. In tumors, blood vessel walls become leaky because of defective vascular architecture, including poorly aligned endothelial cells with wide fenestrations and a lack of a smooth muscle layer. These properties result from rapid angiogenesis or vascularization because tumor cells develop so fast and demand a large supply of nutrients and oxygen [11]. The vascular permeability of tumor tissues can also be enhanced by the actions of secreted factors such as kinin and vascular endothelial growth factor [12]. Furthermore, tumor tissues usually lack effective lymphatic drainage. Therefore, macromolecules or leukocytes can be drained through the leaky blood vessels and be retained. This phenomenon was defined as EPR effect [13]. As a result of such increased vascular permeability, particles ranging from 50 to 500 $\mathrm{nm}$ in size can be selectively delivered to tumor tissue [14]. When these particles are loaded with anticancer drugs, the therapeutics could be selectively delivered to the tumor tissue [15]. In contrast, very small nanoparticles $(<20-30 \mathrm{~nm}$ in diameter $)$ can easily pass through the leaky capillary wall in the tumor but can also be returned to circulating blood by diffusion [16]. Therefore, small particles have good permeability but poor retention. However, after conjugation with a targeting ligand, their retention in the tumor could be greatly enhanced. For tumor targeted drug delivery systems, EPR-effect is now widely accepted as a guiding principle [17].

The size of nanoparticles was found to play a critical role in both the rate and extent of cellular uptake. It was found that 50 $\mathrm{nm}$ transferrin-coated inorganic nanoparticles were taken up by mammalian cells at higher rates and extents compared to smaller and larger sizes in the range of 10-100 nm [18]. The explanation of this optimal size was based on the so-called "wrapping effect" which describes how a cellular membrane encloses nanoparticles. Two factors dictate how fast and how many nanoparticles enter the cellular compartment via "wrapping": the first is the free energy that results from ligand-receptor interaction; the second is the receptor diffusion kinetics onto the wrapping sites on the cellular membrane (Figure 2).

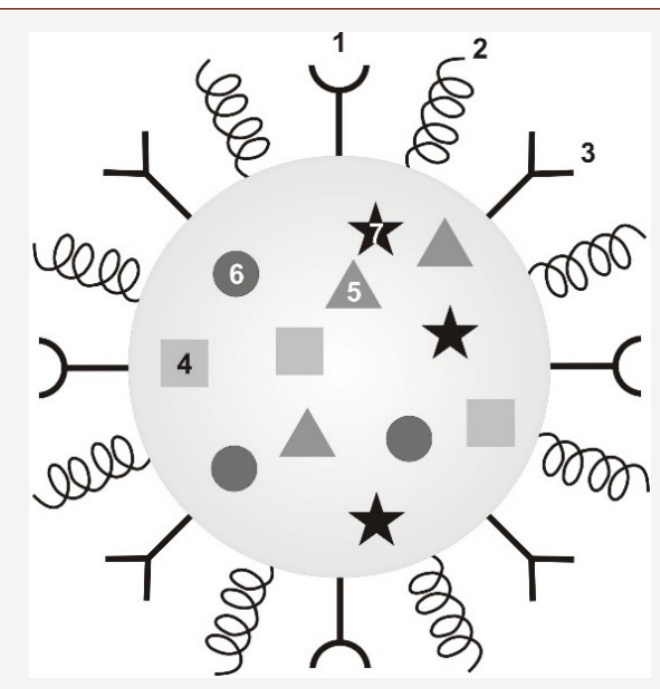

Figure 1: Multifunctional nanoparticle. The following are illustrated: the ability to carry one or more therapeutic agents (1-3) via PEG (2); biomolecular targeting through one or more conjugated antibodies or other recognition agents; imaging signal amplification, by way of coencapsulated contrast agents (4-7) [20].

Considering the contribution of these factors and using mathematical calculations, [19] suggested that nanoparticles with 27-30 nm diameter would have that fastest wrapping time and thus the fastest receptor-mediated endocytosis.

\section{Conclusion}

Cancer appears as a result of various internal and external factors including inherited immune conditions, and genetic mutations and unhealthy diet, and infections, respectively. Angiogenesis and vascularization are well-characterized for tumors. Nanoscale drug delivery platforms have shown promising clinical apprach to treat cancer and inflammatory disorders. Cytotoxic therapeuticsdecorated nanoparticles are used to preferentially kill the rapidly dividing cancer cells while minimizing the damage to the normal tissue. The toxic inorganic nanoparticles are transferred to nontoxic ones by adsorption of functional polymers. After linking to poly (ethylene glycol) molecules, the polymer-coated toxic dots did not change their optical and colloidal properties over a broad range of external environment conditions. This spontaneous encapsulation process removes the problems of particle aggregation in physiological buffers. On the contrary the particles are located at the tumor surroundings due to which therapeutics cellular uptake is increased.

\section{Acknowledgement}

None.

\section{Conflict of Interest}

Author declare no conflict of interest. 


\section{References}

1. Capek I (2017) Noble metal nanoparticles, Preparation, Composite nanostructures, Bio decoration and Collective properties, Series edn. Lockwood DJ (Nanostructure Science and Technology), Capek I (Ed.), Springer, Tokyo, Japan.

2. Shahbazi MA, Hamidi M, Mäkilä EM, Zhang H, Almeida PV, et al. (2013) The mechanisms of surface chemistry effects of mesoporous silicon nanoparticles on immunotoxicity and biocompatibility. Biomaterials 34(31): 7776-7789.

3. Matsumura Y, Maeda, H (1986) A new concept of macromolecular therapies in cancer chemotherapy: mechanism of tumoritropic accumulation of proteins and the antitumor agent SMANCS. Cancer Res 46(12 Pt 1): 6387-6392.

4. Dreher MR, Liu W, Michelich CR, Dewhirst MW, Yuan F, et al. (2006) Tumor vascular permeability, accumulation, and penetration of macromolecular drug carriers. J Natl Cancer Inst 98(5): 335-344.

5. Nomura T, Koreeda N, Yamashita F, Takakura Y, Hashida M (1998) Effect of particle size and charge on the disposition of lipid carriers after intratumoral injection into tissue-isolated tumors. Pharm Res 15(1): 128-132.

6. Hu-Lieskovan S, Heidel JD, Bartlett DW, Davis ME, Triche TJ (2005) Sequence-specific knockdown of EWS-FLI1 by targeted, nonviral delivery of small interfering RNA inhibits tumor growth in a murine model of metastatic Ewing's sarcoma. Cancer Res 65(19): 8984-8992.

7. Sholley MM, Ferguson GP, Seibel HR, Montour JL, Wilson JD (1984) Mechanisms of neovascularization. Vascular sprouting can occur without proliferation of endothelial cells. Lab Invest 51(6): 624-634.

8. Folkman J (2001) Angiogenesis. In: Braunwald E, Fauci AS, Kasper DL (Eds.) Harrison's textbook of internal medicine. $15^{\text {th }}$ edn. McGraw-Hill, New York, USA: 517-530.

9. Folkman J (2007) Angiogenesis: an organizing principle for drug discovery? Nat Rev Drug Discov 6(4): 273-286.
10. Folkman J (1971) Tumor angiogenesis: therapeutic implications. N Engl J Med 285(21): 1182-1186.

11. Hillen F, Griffioen AW (2007) Tumour vascularization: sprouting angiogenesis and beyond. Cancer Metastasis Rev 26(3-4): 489-502.

12. Heldin CH, Rubin K, Pietras K, Ostman A (2004) High interstitial fluid pressure - an obstacle in cancer therapy. Nat Rev Cancer 4(10): 806-813.

13. Maeda H, Wu J, Sawa T, Matsumura Y, Hori K (2000) Tumor vascular permeability and the EPR effect in macromolecular therapeutics: a review. J Control Release 65(1-2): 271-284.

14. Yuan F, Dellian M, Fukumura D, Leunig M, Berk DA, et al. (1995) Vascular permeability in a human tumor xenograft: molecular size dependence and cutoff size. Cancer Res 55(17): 3752-3756.

15. Wang J, Sui M, Fan W (2010) Nanoparticles for tumor targeted therapies and their pharmacokinetics. Current Drug Metabolism 11(12): 129-141.

16. Noguchi Y, Wu J, Duncan R, Strohalm J, Ulbrich K, et al. (1998) Early phase tumor accumulation of macromolecules: a great difference in clearance rate between tumor and normal tissues. Jpn J Cancer Res 89(3): 307-314.

17. Davis ME, Chen ZG, Shin DM (2008) Nanoparticle therapeutics: an emerging treatment modality for cancer. Nat Rev Drug Discov 7(9): 771782.

18. Chithrani BD, Ghazani AA, Chan WCW (2006) Determining the size and shape dependence of gold nanoparticle uptake into mammalian cells. Nano Lett 6(4): 662-668.

19. Gao HJ, Shi WD, Freund LB (2005) Mechanics of receptor mediated endocytosis. Proc Natl Acad Sci USA 102(27): 9469-9474.

20. Ferrari M (2005) Cancer nanotechnology: opportunities and challenges. Nat Rev Cancer 5(3): 161-171. 